\title{
Theory of cosmic ray production in the supernova remnant RX J1713.7-3946
}

\author{
E. G. Berezhko ${ }^{1}$ and H. J. Völk ${ }^{2}$ \\ 1 Yu. G. Shafer Institute of Cosmophysical Research and Aeronomy, 31 Lenin Ave., 677980 Yakutsk, Russia \\ e-mail: berezhko@ikfia.ysn.ru \\ 2 Max Planck Institut für Kernphysik, Postfach 103980, 69029 Heidelberg, Germany \\ e-mail: Heinrich.Voelk@mpi-hd.mpg.de
}

Received 26 November 2005 / Accepted 30 January 2006

\begin{abstract}
Aims. A nonlinear kinetic theory of cosmic ray (CR) acceleration in supernova remnants (SNRs) is employed to investigate the properties of SNR RX J1713.7-3946.

Methods. Observations of the nonthermal radio and X-ray emission spectra as well as the HESS measurements of the very high energy $\gamma$-ray emission are used to constrain the astronomical and the particle acceleration parameters of the system.

Results. Under the assumptions that RX J1713.7-3946 was a core collapse supernova (SN) of type II/Ib with a massive progenitor, has an age of $\approx 1600 \mathrm{yr}$ and is at a distance of $\approx 1 \mathrm{kpc}$, the theory gives indeed a consistent description for all the existing observational data. Specifically it is shown that an efficient production of nuclear CRs, leading to strong shock modification, and a large downstream magnetic field strength $B_{\mathrm{d}} \sim 100 \mu \mathrm{G}$ can reproduce in detail the observed synchrotron emission from radio to X-ray frequencies together with the $\gamma$-ray spectral characteristics as observed by the HESS telescopes. Small-scale filamentary structures observed in nonthermal X-rays provide empirical confirmation for the field amplification scenario which leads to a strong depression of the inverse Compton and Bremsstrahlung fluxes. Going beyond that and using a semi-empirical relation for young SNRs between the resulting CR pressure and the amplified magnetic field energy upstream of the outer SN shock as well as a moderate upper bound for the mechanical explosion energy, it is possible to also demonstrate the actual need for a considerable shock modification in RX J1713.7-3946. It is consistent with RX J1713.7-3946 being an efficient source of nuclear cosmic rays.
\end{abstract}

Key words. cosmic rays - acceleration of particles - supernovae: individual: SNR RX J1713.7-3946 - gamma rays: theory shock waves - X-rays: stars

\section{Introduction}

RX J1713-3946 is a shell-type supernova remnant (SNR), located in the Galactic plane, that was discovered in X-rays with ROSAT (Pfeffermann \& Aschenbach 1996). Further study of this SNR with the ASCA satellite by Koyama et al. (1997) and later by Slane et al. (1999) have shown that the observable X-ray emission is entirely non-thermal, and this property was confirmed in later XMM observations (Cassam-Chenaï et al. 2004).

Given these characteristics it is somewhat surprising that the radio emission is weak. In fact, only part of the shell could be detected in radio synchrotron emission up to now, with a poorly known spectral form (Lazendic et al. 2004).

RX J1713-3946 was also detected in very high energy $\gamma$-rays with the CANGAROO (Muraishi et al. 2000; Enomoto et al. 2002) and HESS (Aharonian et al. 2004, 2006) telescopes. Especially the latter observations show a clear shell structure at $\mathrm{TeV}$ energies which correlates well with the ASCA contours.

This intriguing situation has prompted us to theoretically investigate the acceleration of both electrons and protons in detail, using a nonlinear kinetic theory. It couples the particle acceleration process with the hydrodynamics of the thermal gas (Berezhko et al. 1996; Berezhko \& Völk 2000a). However, in comparison with the other SNRs (SN 1006, Cas A, and Tycho's SNR) that were successfully described within the framework of this theory and finally allowed a prediction of the $\gamma$-ray flux (Berezhko et al. 2002; Völk et al. 2002; Berezhko et al. 2003a,b;
Berezhko \& Völk 2004a; Völk et al. 2005; Ksenofontov et al. 2005), the case of RX J1713.7-3946 puts considerably more obstacles in theory's way. First of all, such decisive astronomical parameters as source distance, present expansion velocity and age are not well known. We shall use the values $d=1 \mathrm{kpc}$ and $t=1612 \mathrm{yr}$ (see below). In addition, the lack of knowledge of the spectral shape of the radio emission makes it difficult to derive - from synchrotron spectral observations - two determining physical quantities: the effective strength of the magnetic field and the proton injection rate into the diffusive acceleration process. This technical difficulty adds to the uncertainty about the type of progenitor star and its possible influence on the circumstellar medium. At first sight it appears therefore impossible to theoretically predict the $\mathrm{TeV} \gamma$-ray emission. We shall argue nevertheless that the observed overall synchrotron spectral shape, from radio frequencies to the X-ray cutoff, and the smallscale filamentary structures in the nonthermal X-ray emission of RX J1713.7-3946 are consistent with efficient CR acceleration associated with a considerably amplified magnetic field. These properties allow in addition a consistent fit of the observed $\mathrm{TeV}$ $\gamma$-ray spectrum. It is strongly dominated by $\pi^{0}$-decay emission.

Assuming that all young SNRs obey a semi-empirical linear correlation between the amplified magnetic field energy density and the pressure of accelerated CRs, and that the mechanical explosion energy of RX J1713.7-3946 has an upper bound of $2.5 \times 10^{51} \mathrm{erg}$, also makes it possible to approximately determine the proton injection rate and thus to predict the time evolution of 
the CR energy content of the SNR and the nonlinear backreaction of the CRs on their own acceleration.

The existence of a compact central object, the X-ray point source 1WGA J1713.4-3949 - most probably a neutron star - and the properties of the observed nonthermal emission of RX J1713.7-3946 strongly indicate that the source is a core collapse supernova (SN) of type II/Ib which exploded into the windblown bubble from a massive progenitor star. This was also argued by Cassam-Chenaï et al. (2004). The main fraction of the remnant volume contains diluted hot bubble gas, whereas at the current evolutionary phase the outer SN shock already propagates into the dense shell of the swept-up ambient interstellar medium (ISM) gas.

The remaining, mainly quantitative uncertainties in the detailed properties of the circumstellar environment still do not allow a unique theoretical solution. Therefore our primary aim is to construct a theoretical model that is consistent in itself and with all available measurements. And this is indeed possible. Nevertheless, we show that also the prediction of a highly efficient nuclear CR production can be cogently argued. In short, we demonstrate that the existing data are consistent with very efficient acceleration of CR nuclei at the SN shock wave which converts a significant fraction of the initial SNR energy content into CR energy. We demonstrate in addition that the available morphological evidence in hard X-rays implies that the leptonic channels of $\gamma$-ray emission (Inverse Compton emission as well as Nonthermal Bremsstrahlung radiation) should be strongly suppressed, and that the calculated hadronic $\gamma$-ray emission is consistent with the HESS spectrum at TeV energies and with the EGRET upper limits in the GeV range. The further going prediction of efficient acceleration of CR nuclei on the basis of two additional semi-empirical assumptions is left to the Discussion section.

\section{Physical parameters of RX J1713-3946}

Following the estimates of Koyama et al. (1997) from ASCA X-ray observations, and of Fukui et al. (2003) from NANTEN $\mathrm{CO}$ line measurements, we adopt a distance $d=1 \mathrm{kpc}$. For the present angular source size of $60^{\prime}$ this leads to a SNR radius $R_{\mathrm{s}} \approx 10 \mathrm{pc}$. Even though a considerably larger distance $d=$ $6 \mathrm{kpc}$ to the SNR RX J1713.7-3946, estimated in earlier studies (Slane et al. 1999), is still being discussed (e.g. Cassam-Chenai et al. 2004; Hiraga et al. 2005), the recent study of molecular clouds toward SNR RX J1713.7-3946 (Moriguchi et al. 2005) "strongly supports" the distance of $1 \mathrm{kpc}$.

A rather simple theoretical consideration shows that the SNR should be quite young in order to reach such a large size and to be at the same time such a bright source of nonthermal $\mathrm{X}$-rays. The observed energy flux of nonthermal X-rays at en$\operatorname{ergy} \epsilon_{v}=4 \mathrm{keV}$ is $v S_{v} \approx 100 \mathrm{eV} /\left(\mathrm{cm}^{2} \mathrm{~s}\right.$ ) (Slane et al. 1999) which for the distance $d=1 \mathrm{kpc}$ translates into the synchrotron luminosity $v L_{v}=4 \pi d^{2} v S_{v} \approx 2 \times 10^{34} \mathrm{erg} / \mathrm{s}$. Here $v$ is the emission frequency, $L_{v}$ and $S_{v}$ are the spectral luminosity and integral flux, measured at the distance $d$, respectively. According to the theoretical estimates of Berezhko \& Völk (2004b) such a high luminosity is possible only if the shock speed is not very low, $V_{\mathrm{s}}>1500 \mathrm{~km} \mathrm{~s}^{-1}$. Otherwise the electron cutoff energy becomes so small that it implies a very low X-ray luminosity. This leads to a restriction on the SNR age for an assumed uniform external ISM. In such a case the present epoch of SNR expansion is described by the Sedov solution:

$R_{\mathrm{S}}=\left(1.2 \times 10^{24} E_{\mathrm{sn}} / N_{\mathrm{ISM}}\right)^{1 / 5} t^{2 / 5}$, where $E_{\mathrm{sn}}$ is the $\mathrm{SN}$ explosion energy, $N_{\mathrm{ISM}}=\rho_{\mathrm{ISM}} / m_{\mathrm{p}}$ is the interstellar medium (ISM) number density, $\rho_{\text {ISM }}$ is the ISM density and $m_{\mathrm{p}}$ is the proton mass. Since the shock radius and the shock speed are related as $V_{\mathrm{s}}=0.4 R_{\mathrm{S}} / t$ the above condition gives a restriction for the SNR age $t<2.7 \times$ $10^{3} \mathrm{yr}$. If we assume the standard value $E_{\mathrm{sn}}=10^{51} \mathrm{erg}$ for the SN explosion energy and use the observed SNR radius $R_{\mathrm{S}}=10 \mathrm{pc}$, Eq. (1) will give us $N_{\mathrm{ISM}}<0.3 \mathrm{~cm}^{-3}$. In such a diluted ISM the maximum possible $\mathrm{TeV} \gamma$-ray flux $\epsilon F_{\gamma}(\epsilon)=10\left(N_{\text {ISM }} / 1 \mathrm{~cm}^{-3}\right)(d / 1 \mathrm{kpc})^{-2} \mathrm{eV} \mathrm{cm}^{-2} \mathrm{~s}^{-1}$ (Berezhko \& Völk 2000b) and the expected nonthermal X-ray flux $v L_{v}=$ $10^{33}\left(K_{\text {ep }} / 10^{-4}\right)$ erg/s (Berezhko \& Völk 2004b) would be significantly below the observed values. Here $K_{\mathrm{ep}}$ denotes the electron:proton ratio in the energetic particle population.

This contradiction can be resolved if SNR RX J1713.7-3946 is the result of a core collapse $\mathrm{SN}$ of type $\mathrm{II} / \mathrm{Ib}$ which exploded into the adiabatic, very diluted bubble created by the wind of a massive progenitor star. This is also required by the probable existence of a compact remnant in the center, the neutron star 1WGA J1713.4-3949 (e.g. Cassam-Chenaï et al. 2004). According to stellar wind theory (e.g. Weaver et al. 1977; Chevalier 1982), the bubble is bounded by a dense, massive shell which consists of the swept-up, cooled ISM material. Note that Slane et al. (1999), Fukui et al. (2003) and Cassam-Chenaï et al. (2004) came to the same conclusion based on different types of arguments (see also Ellison et al. 2001). In a very general study the combined SNR dynamics and diffusive shock acceleration in wind-SNe has been investigated by Berezhko \& Völk (2000b).

Progenitor stars of core collapse $\mathrm{SNe}$, which have intense winds, are massive main-sequence stars with initial masses $M_{\mathrm{i}}>15 M_{\odot}$ (e.g. Abbot 1982). In the mean, during their evolution in the surrounding uniform ISM of gas number density $\rho_{0}=m_{\mathrm{p}} N_{\text {ISM }}$, they create a bubble of size (Weaver et al. 1977; Chevalier \& Liang 1989)

$R_{\mathrm{sh}}=0.76\left(0.5 \dot{M} V_{\mathrm{w}}^{2} t_{\mathrm{w}}^{3} / \rho_{0}\right)^{1 / 5}$,

where $\dot{M}$ is the mass-loss rate of the progenitor, $V_{\mathrm{w}}$ is the wind speed, and $t_{\mathrm{w}}$ is the duration of the wind period. This bubble could be adiabatic, and then have very low gas density, or it could be modified by mixing of the hot bubble gas with surrounding shell material. The large size of RX J1713.7-3946 and its low age require an adiabatic bubble, which we shall adopt for the sequel.

In order to determine the $\mathrm{SN}$ shock dynamics inside the shell we model the gas number density distribution $N_{\mathrm{g}}$ as a constant in the bubble and as a power law in radius in the shell. This leads to the following radial profile

$N_{\mathrm{g}}=N_{\mathrm{b}}+\left(r / R_{\mathrm{sh}}\right)^{3\left(\sigma_{\mathrm{sh}}-1\right)} N_{\mathrm{sh}}$,

where $N_{\text {sh }}=\sigma_{\text {sh }} N_{\text {ISM }}$ is the peak number density in the shell, $N_{\mathrm{b}}$ is the gas number density inside the bubble, typically very small compared with the shell density, and $\sigma_{\text {sh }}=N_{\text {sh }} / N_{\text {ISM }}$ is the shell compression ratio. We emphasize that the compression ratio $\sigma_{\text {sh }}$ can exceed the adiabatic upper limit of 4 as a result of the radiative cooling in the shell.

The mass of the bubble

$M_{\mathrm{b}}=\left(4 \pi R_{\mathrm{sh}}^{3} / 3\right) m_{\mathrm{p}} N_{\mathrm{b}}$

is rather small, $M_{\mathrm{b}}<M_{\odot}$, in the case of moderate progenitor masses $M_{i}<20 M_{\odot}$ (e.g. Chevalier \& Liang 1989), whereas the shell mass

$M_{\mathrm{sh}}=4 \pi N_{\mathrm{sh}} m_{\mathrm{p}} \int_{0}^{R_{\mathrm{sh}}} \mathrm{d} r r^{2}\left(r / R_{\mathrm{sh}}\right)^{3(\sigma-1)}=\left(4 \pi R_{\mathrm{sh}}^{3} / 3\right) N_{\mathrm{ISM}} m_{\mathrm{p}}$

amounts to a few thousand solar masses (see below). 
During SNR shock propagation through the adiabatic bubble, only a small fraction of the mechanical explosion energy is therefore given to gas of stellar origin. The main fraction of the explosion energy is deposited in the shell.

Here we use the gas number density distribution $N_{\mathrm{g}}(r)=$ $\rho(r) / m_{\mathrm{p}}$ in the form

$N_{\mathrm{g}}=0.008+1.1[\mathrm{r} /(10 \mathrm{pc})]^{12} \mathrm{~cm}^{-3}$

which gives a consistent fit for all existing data for SNR RX J1713.7-3946. Such a distribution corresponds to a bubble with $\sigma_{\mathrm{sh}}=5, N_{\mathrm{b}}=0.008 \mathrm{~cm}^{-3}$, and a range $17.6<R_{\mathrm{sh}}<$ $19.9 \mathrm{pc}$, created by the wind of a main-sequence star of initial mass $15 M_{\odot}<M_{i}<20 M_{\odot}$ in a surrounding ISM of number density $110<N_{\text {ISM }}<500 \mathrm{~cm}^{-3}$, respectively (Chevalier $\&$ Liang 1989). Such a high ISM density, required to produce a bubble of such a small size, is quite consistent with the value $N_{\text {ISM }}=300 \mathrm{~cm}^{-3}$ derived from the analysis of the X-ray emission of SNR RX J1713.7-3946 (Cassam-Chenaï et al. 2004). This implies that the bubble is formed inside a molecular cloud; a discussion of the $\mathrm{CO}$ emission from the neighbourhood of the SNR is given in the paper by Fukui et al. (2003).

For the SNR age we use the value $t=1612 \mathrm{yr}$, which is consistent with the hypothesis of Wang et al. (1997), based on historical Chinese records, that RX J1713-3946 is the remnant of the AD393 "guest star".

We adopt the following values of SNR parameters: $E_{\mathrm{sn}}=$ $1.8 \times 10^{51} \mathrm{erg}, M_{\mathrm{ej}}=3.5 M_{\odot}$. As shown below, these parameter values lead to a good fit for the observed SNR properties. For a better determination of the explosion energy we would need the value of the shock speed $V_{\mathrm{s}}$ which is unfortunately is not measured.

\subsection{Magnetic field strength}

Whereas the above physical parameters - explosion type, explosion energy, progenitor mass loss, circumstellar density, age and distance - are part of an internally consistent picture whose detailed quantitative specification allows a consistent fit to the observed X-ray synchrotron and $\gamma$-ray emission, the magnetic field strength plays a specific role in this discussion. The reason is that its strength decides the relative role of electrons and nuclear particles in the $\gamma$-ray emission. In the following we shall therefore go into the question of the field determination in some detail.

As indicated in the Introduction, there are two obvious ways to determine the effective magnetic field in a SNR, and we have used both of them in the past (Völk et al. 2005; Berezhko 2005) for SN 1006, Cas A, and Tycho's SNR.

The first method analyses the overall synchrotron spectrum. If, first of all, the radio continuum spectrum is softer than the test particle limit, i.e. with a photon index larger than $\alpha=0.5$, then this is attributed to the radiation of electrons that have been accelerated at the subshock of an overall nonlinearly modified shock. This requires (i) an effective magnetic field strength which is above a certain limit and (ii) that the electrons have energies below $1 \mathrm{GeV}$, where their spectrum is steeper than $N_{\mathrm{e}} \propto p^{-2}$, in order to obtain such a steep synchrotron spectrum (see Berezhko et al. 2003b; Berezhko 2005, for details). In addition, the form of the X-ray synchrotron spectrum, including its cutoff, must be consistent with this magnetic field strength. This determines the effective field strength inside the SNR to within about 30 percent. In all cases considered in the past the field turned out to be amplified by a factor between 5 and 10 relative to the expected upstream field strength (Völk et al. 2005).
The second method is also empirical and makes use of the synchrotron morphology of the SNR at keV energies (Berezhko \& Völk 2004a; Völk et al. 2005). Interpreting the thickness of sharp filaments in hard X-rays - typically in the line-free energy region between 4 and $6 \mathrm{keV}$ - as the result of synchrotron losses during and after the electron acceleration process, we obtain an independent measure of the downstream magnetic field strength. Within an accuracy of 20 to 30 percent this field strength agrees with that determined by the first method.

The present status of the radio synchrotron measurements for SNR RX J1713.7-3946 does not determine the radio spectral index, and therefore we have only limited restrictions on the field strength. Fortunately, however, the synchrotron morphology gives a rather definite measure of the field. The recent XMM observations of Hiraga et al. (2005) show a radial X-ray profile (see their Figs. 1 and 2) which we interpret to show unequivocally the filamentary structure behind the outer SNR shock. The exponential angular width of the filament corresponds to 5.1 bins, of width $\Delta \psi=25.6^{\prime \prime}$ each (Hiraga, private communication), within the radial profile shown by Hiraga et al. (2005). Therefore the profile width corresponds to $\Delta \psi \approx 2.2^{\prime}$, or to a spatial scale $L=2 \times 10^{18} \mathrm{~cm}$ at a distance of $1 \mathrm{kpc}$.

As it was already demonstrated for other young SNRs (Berezhko et al. 2003a; Berezhko \& Völk 2004a; Völk et al. 2005), the measured width of the projected radial profile of the nonthermal X-ray emission determines the internal (downstream) magnetic field according to the expression

$B_{\mathrm{d}}=\left[3 m_{\mathrm{e}}^{2} c^{4} /\left(4 e r_{0}^{2} l_{2}^{2}\right)\right]^{1 / 3}\left(\sqrt{1+\delta^{2}}-\delta\right)^{-2 / 3}$,

where $\delta^{2}=0.12\left[c /\left(r_{0} v\right)\right]\left[V_{\mathrm{s}} /(\sigma c)\right]^{2}, l_{2} \approx L / 7$ is the radial width of the X-ray emissivity $q_{v}\left(\epsilon_{v}, r\right), r_{0}$ is the classical electron radius and $\sigma$ is the total shock compression ratio. Substituting into this expression the values $L=2 \times 10^{18} \mathrm{~cm}, \sigma=6.3$ (see below), $V_{\mathrm{s}}=1800 \mathrm{~km} \mathrm{~s}^{-1}$, and $v=1.8 \times 10^{17} \mathrm{~Hz}\left(\epsilon_{v}=0.7 \mathrm{keV}\right)$, we obtain $B_{\mathrm{d}} \approx 65 \mu \mathrm{G}$.

Given the finite resolution used to analyse these XMM data, which leads to a wider profile than the actual profile (see Fig. 5 below), this value for $B_{\mathrm{d}}$ is clearly a lower limit to the true amplified field. However, the Hiraga et al. (2005) XMM profile has the great advantage that it certainly corresponds to the outer shock, whereas for the much narrower $20^{\prime \prime}$ Chandra profile measured by Uchiyama et al. (2003), as analysed in Völk et al. (2005), the doubt remained, if that profile was due to the outer shock or rather due to some other discontinuity in the SNR plasma. On the other hand, there is at present only one such profile available, and we are left with the question as to how representative this profile and the derived field value is. From general gas dynamics considerations we argue that the probability is extremely low that the XMM profile corresponds to a singular point at the shock surface. We therefore conclude that a lower limit to the magnetic field strength at the outer shock is $65 \mu \mathrm{G}$. Extrapolating from the $20^{\prime \prime}$ scale of the Chandra profile, the lower field limit could be as large as $230 \mu \mathrm{G}$. Examinations of other XMM or Chandra radial profiles are expected to confirm this conclusion.

Below we shall derive the effective field strength as a result of fitting the synchrotron spectrum, in particular its X-ray part. For other SNRs this fit and the filament sizes give consistent results for the effective field. It will be shown that the spectral fit can be achieved with values $B_{\mathrm{d}}=126 \mu \mathrm{G}$ as well as $B_{\mathrm{d}}=250 \mu \mathrm{G}$. These values roughly lie in between the two extremes discussed above. Such values of $B_{\mathrm{d}}$ are significantly higher than typical ambient magnetic fields, also for the considered bubble wall in the molecular cloud (the density at the shock 
is still a factor $\sim 100$ lower than in the cloud which implies a strongly decompressed field upstream of the SNR shock compared to the value in the cloud). It must be attributed to field amplification at the shock front due to the strong wave production by the acceleration of CRs far into the nonlinear regime (Bell \& Lucek 2001; Bell 2004).

\section{Model}

Hydrodynamically, a core collapse supernova (SN) explosion ejects a shell of matter with total energy $E_{\mathrm{sn}} \approx 10^{51} \mathrm{erg}$ and mass $M_{\mathrm{ej}}$ equal to a few solar masses. During an initial period the shell material has a broad distribution in velocity $v$. The fastest part of this ejecta distribution can be described by a power law $\mathrm{d} M_{\mathrm{ej}} / \mathrm{d} v \propto v^{2-\mathrm{k}}$ (e.g. Jones et al. 1981; Chevalier 1982). The interaction of the ejecta with the interstellar medium (ISM) creates a strong shock there which heats the thermal gas and accelerates particles diffusively to a nonthermal CR component of comparable energy density. For core collapse $\mathrm{SN}$ explosions a value $k=8$ appears appropriate which we adopt in this paper.

As pointed out in the Introduction, our theory for this process is based on a fully time-dependent, spherically symmetric solution of the CR transport equations, coupled nonlinearly with the gas dynamic equations for the thermal gas component. Since all relevant equations, initial and boundary conditions for this model have already been described in detail in these papers, we do not present them here and only briefly discuss the most important aspects below (reviewed also by Völk 2003; and Berezhko 2005).

The coupling between the ionised thermal gas (plasma) and the energetic particles occurs primarily through magnetic field fluctuations carried by the plasma which scatter energetic particles in pitch angle and energize them, especially in shock waves. The plasma physics of the field fluctuations is not worked out yet in full detail. However the accelerating CRs effectively excite magnetic fluctuations upstream of the outer SN shock in the form of Alfvén waves (e.g. Bell 1978; Blandford \& Ostriker 1978) as a result of the streaming instability. In quasilinear approximation the wave amplitudes $\delta B$ grow to very high amplitudes $\delta B>B$, where $B$ is the average field strength (McKenzie \& Völk 1982). Since these fluctuations scatter CRs extremely strongly, the CR diffusion coefficient is assumed to be as small as the Bohm limit $\kappa(p)=\kappa(m c)(p / m c)$, where $\kappa(m c)=m c^{3} /(3 e B), e$ and $m$ are the particle charge and mass, $p$ denotes the particle momentum, and $c$ is the speed of light.

If $B_{\text {ISM }}$ is the pre-existing field in the surrounding medium, then the strong streaming instability would suggest that the instability growth is restricted by some nonlinear mechanism to the level $\delta B \sim B_{\text {ISM }}$, any further turbulent energy being dissipated into the thermal gas (Völk \& McKenzie 1981). The early attempts to give a full nonlinear description of the magnetic field evolution in a numerical simulation (Lucek \& Bell 2000; Bell \& Lucek 2001) concluded that a considerable amplification of the "average" magnetic field in the smooth shock precursor - produced by the finite CR pressure - should occur. Broadly speaking, it was expected that a non-negligible fraction of the shock ram pressure $\rho_{0} V_{\mathrm{s}}^{2}\left(\rho_{0}\right.$ is the ambient gas density at the current $\mathrm{SN}$ shock position) is converted into magnetic field energy. Subsequently, Bell (2004) argued that this amplification is the result of a nonresonant instability, giving rise to what we shall call the effective upstream field $B_{0}>B_{\mathrm{ISM}}$, on top of which the Alfvén waves grow to amplitudes $\delta B \sim B_{0}$. The Bohm limit and the gas heating are then to be calculated with $B=B_{0}$.
In our analyses of the synchrotron spectrum of SN 1006, Tycho's SNR, and Cas A, such magnetic field amplifications were indeed found; they can only be produced as a nonlinear effect by a very efficiently accelerated nuclear CR component. Its energy density, consistent with all existing data, is so high that it is able to strongly excite magnetohydrodynamic fluctuations, and thus to amplify the upstream magnetic field $B_{\mathrm{ISM}}$ to an effective field $B_{0}>B_{\text {ISM }}$, at the same time to permit efficient $\mathrm{CR}$ scattering on all scales, reaching the Bohm limit. The same large effective magnetic field turns out to be required, within the errors, by the comparison of this self-consistent theory with the morphology of the observed X-ray synchrotron emission, in particular, its spatial fine structure. We shall therefore also here allow for the possibility of an amplified field.

The number of suprathermal protons injected into the acceleration process is described by a dimensionless injection parameter $\eta \ll 1$ which is a fixed fraction of the gas particles entering the shock front. For simplicity it is assumed that the injected particles have a velocity four times higher than the postshock sound speed. We have argued before that ion injection is quite efficient at the quasiparallel portions of the shock surface, where it is characterised by values $\eta=10^{-4}$ to $10^{-3}$ (see Völk et al. 2003, for details). Since this injection is expected to be strongly suppressed at the quasiperpendicular part of the shock, one should renormalize the results for the nucleonic spectrum, calculated within the spherically symmetric model. The lack of symmetry in the actual SNR can be approximately taken into account by a renormalization factor $f_{\text {re }}<1$, roughly $f_{\text {re }}=0.15$ to 0.25 , which diminishes the nucleonic CR production efficiency, calculated in the spherical model, and all effects associated with it.

We assume that also electrons are injected into the acceleration process at the shock front. Formally their injection momentum is taken to be the same as that of the protons. Since the details of the electron injection process are poorly known, we chose the electron injection rate such that the electron:proton ratio $K_{\text {ep }}$ (which we define as the ratio of their distribution functions at all rigidities where the protons are already relativistic and the electrons have not been yet cooled radiatively) is a constant to be determined from the synchrotron observations. It is demonstrated below, that in the case of SNR RX J1713.7-3946 the scarce existing data nevertheless give a possibility to estimate the values of $B_{0}, \eta$ and $K_{\text {ep }}$.

The electron dynamics is exactly the same as that for protons for electron rigidities corresponding to ultrarelativistic protons, as long as synchrotron losses are neglected. Therefore, beyond such rigidities and below the loss region the distribution function of accelerated electrons has the form $f_{\mathrm{e}}(p)=K_{\mathrm{ep}} f(p)$ at any given time. The electron distribution function $f_{\mathrm{e}}(p)$ deviates only at sufficiently large momenta from this relation due to synchrotron losses, which are taken into account by supplementing the ordinary diffusive transport equation by a radiative loss term.

Clearly, from the point of view of injection/acceleration theory, we must treat $K_{\mathrm{ep}}$, together with $B_{0}$ and $\eta$, as a theoretically not very well constrained parameter to be quantitatively determined by comparison with synchrotron observations to the extent that they are available.

The solution of the dynamic equations at each instant of time yields the CR spectrum and the spatial distributions of CRs and thermal gas. This allows the calculation of the expected fluxes of nonthermal emission produced by the accelerated CRs. 


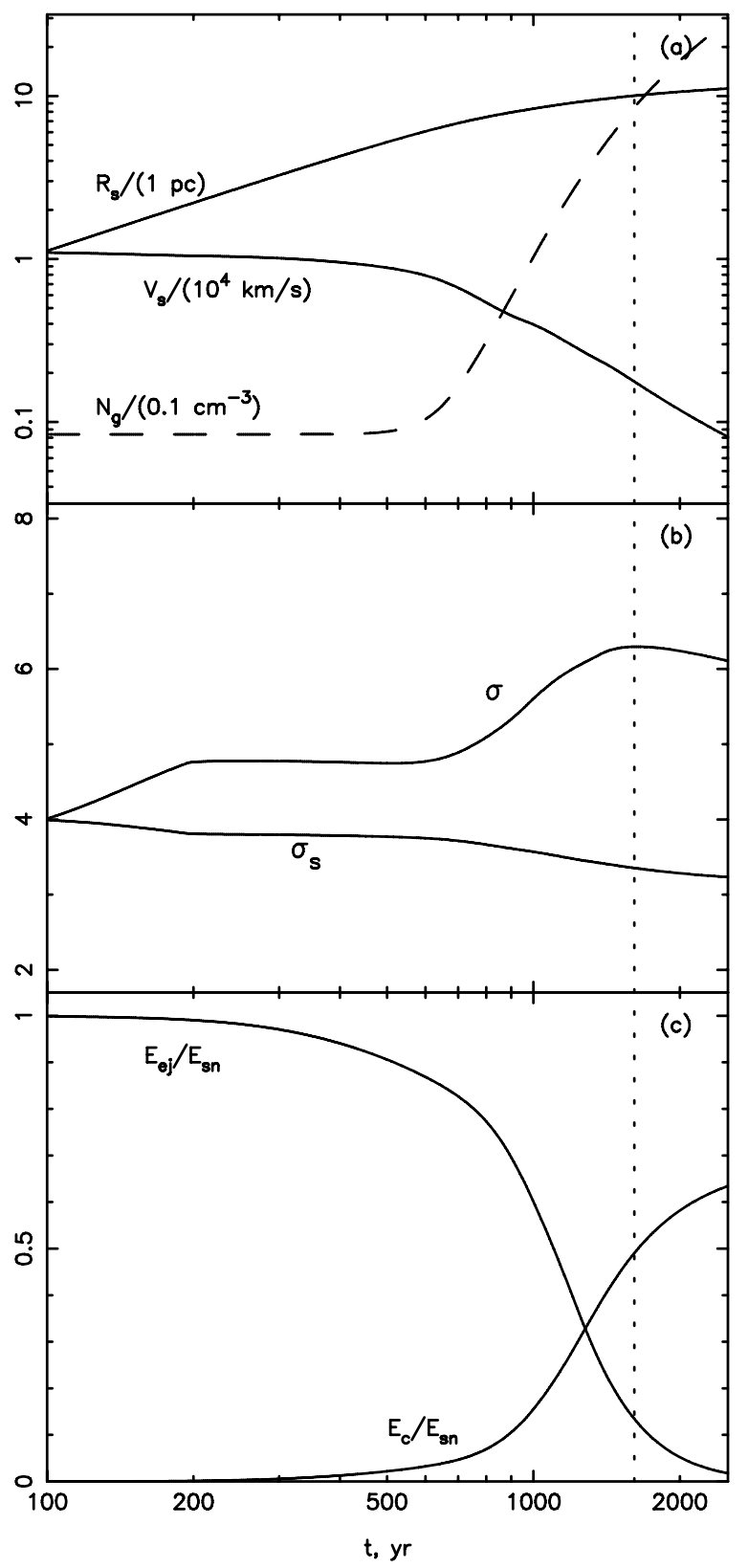

Fig. 1. a) Shock radius $R_{\mathrm{s}}$ and shock speed $V_{\mathrm{s}}$; b) total shock $(\sigma)$ and subshock $\left(\sigma_{\mathrm{s}}\right)$ compression ratios; c) ejecta $\left(E_{\mathrm{ej}}\right)$ and CR $\left(E_{\mathrm{c}}\right)$ energies as a function of time, normalised to the total mechanical SNR energy $E_{\mathrm{sn}}$. The vertical dotted line marks the current evolutionary epoch.

\section{Results}

The computed gas dynamical characteristics of the SNR are shown in Fig. 1. They demonstrate in Fig. 1a that the calculation fits the observed SNR size $R_{\mathrm{s}}$ for the assumed distance and SNR age. The resulting shock velocity is $V_{\mathrm{s}} \approx 1840 \mathrm{~km} \mathrm{~s}^{-1}$.

To obtain a good fit for the observed synchrotron and gamma-ray spectra (see below) we assume a proton injection rate $\eta=3 \times 10^{-4}$. We want to emphasise that the most reliable way to determine $\eta$ is based on the measured spectral shape of the radio emission. Unfortunately this cannot be done for the case of RX J1713.7-3946, because the shape of the radio spectrum is poorly known. The assumed injection rate is nevertheless in the same range as in other remnants, where this quantity can indeed be directly determined. It leads to a significant

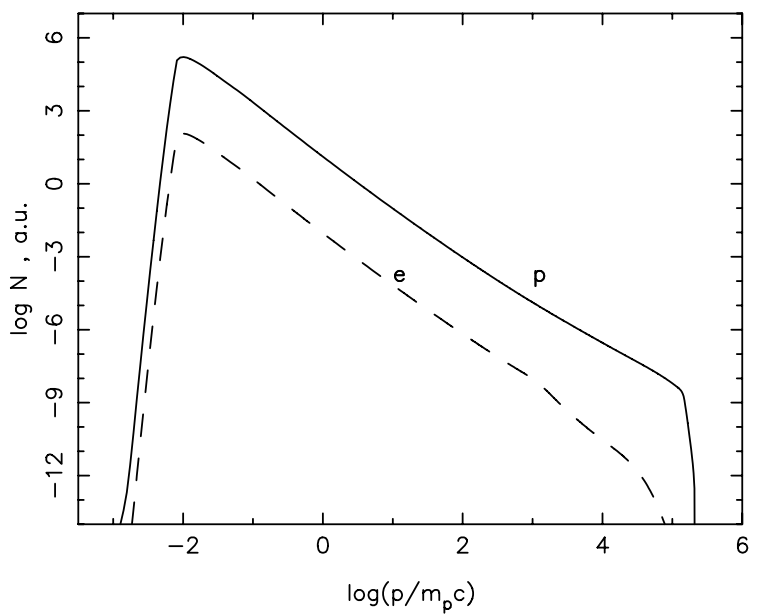

Fig. 2. Spatially integrated CR spectrum as function of particle momentum. Solid and dashed lines correspond to protons and electrons, respectively.

nonlinear modification of the shock which, at the current age of $t=1612 \mathrm{yr}$, has a total compression ratio $\sigma=6.3$ and a subshock compression ratio $\sigma_{\mathrm{s}}=3.3$ (Fig. 1b). In the present case the shape of the TeV-spectrum (see below) is the only spectral characteristic which furnishes evidence that the SN shock is strongly modified.

We note here that the lack of a restriction on the injection rate, resulting from the lack of knowledge of the radio spectral index, facilitates the fit of the spectrum. However, we will argue in Sect. 5 that no basically different injection rate is possible.

For its adopted density the hot wind bubble contains only a small amount of gas $M_{\mathrm{b}} \approx 0.3 M_{\odot}$. This is consistent with the range of $0.07<M_{\mathrm{b}}<0.6 M_{\odot}$ for the initial stellar masses $M_{\mathrm{i}}$ in the range $15 M_{\odot}<M_{\mathrm{i}}<20 M_{\odot}$. Due to this fact the SN shock has deposited less than $20 \%$ of the explosion energy while propagating through the bubble $(t<700 \mathrm{yr})$, as seen from Fig. 1c. Yet at the current epoch the SN shock has already swept up a considerable mass $M_{\mathrm{sw}} \approx 20 M_{\odot}$ and therefore the ejecta has already transformed about $90 \%$ of its initial energy into thermal gas and CRs.

In spherical symmetry the acceleration process is then characterised by a high efficiency: at the current time about $53 \%$ of the explosion energy have been transferred to CRs, and the $\mathrm{CR}$ energy content $E_{\mathrm{c}}$ continues to increase to a maximum of about $60 \%$ in the later phase (Fig. 1c), when the highest energy particles start to leave the source. As typically predicted by the spherically symmetric model, such a CR acceleration efficiency is significantly higher than required for the average replenishment of the Galactic CRs by SNRs, corresponding to $E_{\mathrm{c}} \approx 0.1 E_{\mathrm{sn}}$. And as discussed above, the necessary deviation of the SNR from spherical symmetry requires a renormalization of the number of hadronic CRs. We choose the value $f_{\text {re }}=1 / 5$.

With this renormalization the CRs inside RX J1713-3946 SNR already contain

$E_{\mathrm{c}}=0.53 f_{\mathrm{re}} E_{\mathrm{sn}} \approx 2 \times 10^{50} \mathrm{erg}$.

The volume-integrated (or overall) CR spectrum

$N(p, t)=16 \pi^{2} p^{2} \int_{0}^{\infty} \mathrm{d} r r^{2} f(r, p, t)$

has, for the case of protons, almost a pure power-law form $N \propto p^{-\gamma}$ over a wide momentum range from $10^{-2} m_{\mathrm{p}} c$ up to the 


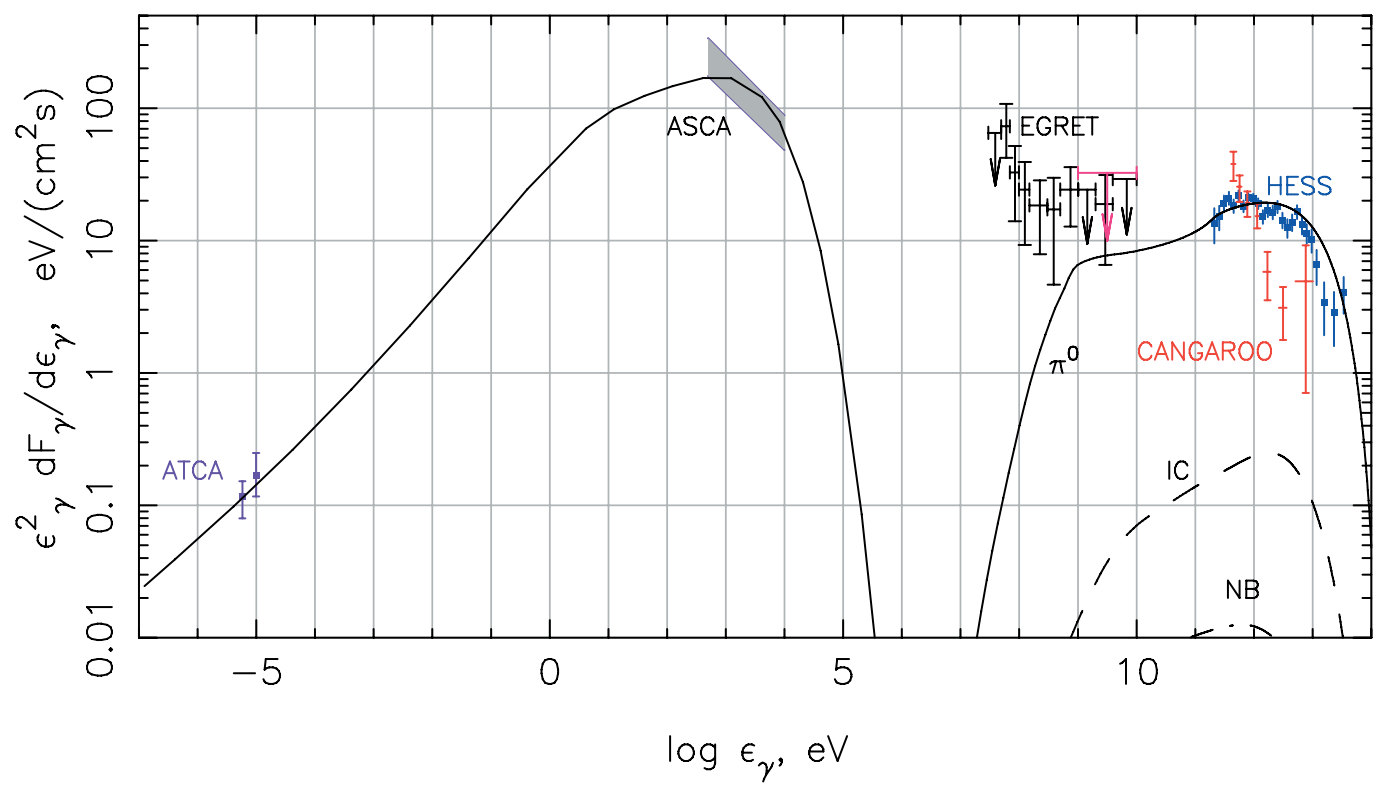

Fig. 3. Spatially integrated spectral energy distribution of RX J1713.7-3946. The ATCA radio data (cf. Aharonian et al. 2006), ASCA X-ray data (cf. Aharonian et al. 2006), EGRET spectrum of 3EG J1714-3857 (Reimer \& Pohl 2002), CANGAROO data (Enomoto et al. 2002), in red color) and HESS data (Aharonian et al. 2006), in blue color) are shown. The EGRET upper limit for the RX J1713.7-3946 position (Aharonian et al. 2006) is shown as well (red colour). The solid curve at energies above $10^{7} \mathrm{eV}$ corresponds to $\pi^{0}$-decay $\gamma$-ray emission, whereas the dashed and dash-dotted curves indicate the inverse Compton (IC) and Nonthermal Bremsstrahlung (NB) emissions, respectively.

cutoff momentum $p_{\max } \approx 2 \times 10^{5} m_{\mathrm{p}} c$ (Fig. 2). This value $p_{\max }$ is limited mainly by the finite size and speed of the shock, its deceleration and the adiabatic cooling effect in the downstream region (Berezhko 1996). As a result of the shock modification, the power-law index slowly varies from $\gamma=2.2$ at $p \lesssim m_{\mathrm{p}} c$ to $\gamma=1.7$ at $p \gtrsim 10^{3} m_{\mathrm{p}} c$.

The shape of the overall electron spectrum $N_{\mathrm{e}}(p)$ deviates from that of the proton spectrum $N(p)$ at high momenta $p>$ $p_{1} \sim 10^{3} m_{\mathrm{p}} c$, on account of the synchrotron losses during their residence time in the downstream region with a magnetic field strength $B_{\mathrm{d}} \approx 130 \mu \mathrm{G}$ which is assumed uniform in this region $\left(B_{\mathrm{d}}=B_{2}=\sigma B_{0}\right)$. Cf. Berezhko et al. (2002) the synchrotron losses become important for electron momenta greater than

$\frac{p_{1}}{m_{\mathrm{p}} c} \approx 1.3\left(\frac{10^{8} \mathrm{yr}}{t}\right)\left(\frac{10 \mu \mathrm{G}}{B_{\mathrm{d}}}\right)^{2}$,

where $p_{1}$ is relevant only in the evolutionary stage, when it becomes lower than the electron cutoff momentum $p_{\max }^{\mathrm{e}}, p_{1}<p_{\max }^{\mathrm{e}}$ This is already the case at present. Substituting the SN age $t=1612 \mathrm{yr}$ into this expression, we have $p_{1} \approx 500 \mathrm{~m}_{\mathrm{p}} c$, in agreement with the numerical results (Fig. 2).

The shock continuously produces an electron spectrum $f_{\mathrm{e}} \propto$ $p^{-q}$, with $q \approx 4$, up to the maximum momentum $p_{\max }^{\mathrm{e}}(t)$ which is at the present time already much larger than $p_{1}$. Therefore, within the momentum range $p_{1}<p<p_{\max }^{\mathrm{e}}$, the electron spectrum is considerably steeper $N_{\mathrm{e}} \propto p^{-3}$ due to synchrotron losses taking place in the downstream region after the acceleration at the shock front.

For $p_{\max }^{\mathrm{e}}<p_{\max }$ the maximum electron momentum can be estimated by equating the synchrotron loss time and the acceleration time. This gives (e.g. Berezhko et al. 2002):

$$
\begin{aligned}
\frac{p_{\max }^{\mathrm{e}}}{m_{\mathrm{p}} c}= & 6.7 \times 10^{4}\left(\frac{V_{\mathrm{s}}}{10^{3} \mathrm{~km} \mathrm{~s}^{-1}}\right) \\
& \times \sqrt{\frac{(\sigma-1)}{\sigma\left(1+\sigma^{2}\right)}\left(\frac{10 \mu \mathrm{G}}{B_{0}}\right)} .
\end{aligned}
$$

At the current epoch $V_{\mathrm{s}} \approx 1840 \mathrm{~km} \mathrm{~s}^{-1}$ which leads to a maximum electron momentum $p_{\max }^{\mathrm{e}} \approx 1.3 \times 10^{4} m_{\mathrm{p}} c$, in agreement with the numerical results (Fig. 2).

We note that during the last thousand years the SN shock speed has been going down rapidly. In previous evolutionary epochs it has therefore produced electron spectra with cutoff momenta $p_{\max }^{\mathrm{e}}$ larger than that of the current epoch. Due to this fact the overall electron spectrum has a relatively smooth cutoff, extending up to $p \sim 10^{5} m_{\mathrm{p}} c$ (see Fig. 2). Together with the synchrotron cooling it provides a very good fit of the observed $\mathrm{X}$-ray spectrum (see below).

The parameters $K_{\mathrm{ep}} \approx 10^{-4}$ and $B_{\mathrm{d}}=126 \mu \mathrm{G}$ give good agreement between the calculated and the measured synchrotron emission in the radio to X-ray ranges (Fig. 3). The steepening of the electron spectrum at high energies $>10^{3} m_{\mathrm{p}} c^{2}$ due to synchrotron losses and the smooth cutoff of the overall electron spectrum naturally yield a fit to the X-ray data with their soft spectrum.

The overall broadband spectral energy distribution is displayed in Fig. 3, together with the experimental data from ATCA at radio wavelengths, as estimated for the full remnant by Aharonian et al. (2006), the X-ray data from ASCA, the EGRET spectrum of the nearby source 3EG J1714-3857 (Reimer \& Pohl 2002), and the TeV $\gamma$-ray spectra from CANGAROO (Enomoto et al. 2002) and HESS (Aharonian et al. 2006). The data also include the more recent EGRET upper limit based on the assumption that 3EG J1714-3857 is not physically associated with RX J1713.7-3946. The overall fit is impressive, noting that the choice of a few key parameters like $\eta, B_{\mathrm{d}}$, and $E_{\mathrm{sn}}$ in the theory allows a spectrum determination over more than 19 decades. The remainder of this section will give a detailed discussion of these spectra and of the morphology.

In Fig. 4 we separately present the synchrotron spectrum, produced at the current epoch by the accelerated electrons. For comparison we also include (through the dashed curve) a synchrotron spectrum, which would correspond to an artificial scenario with a proton injection rate so small $\left(\eta=10^{-5}\right)$ that the 


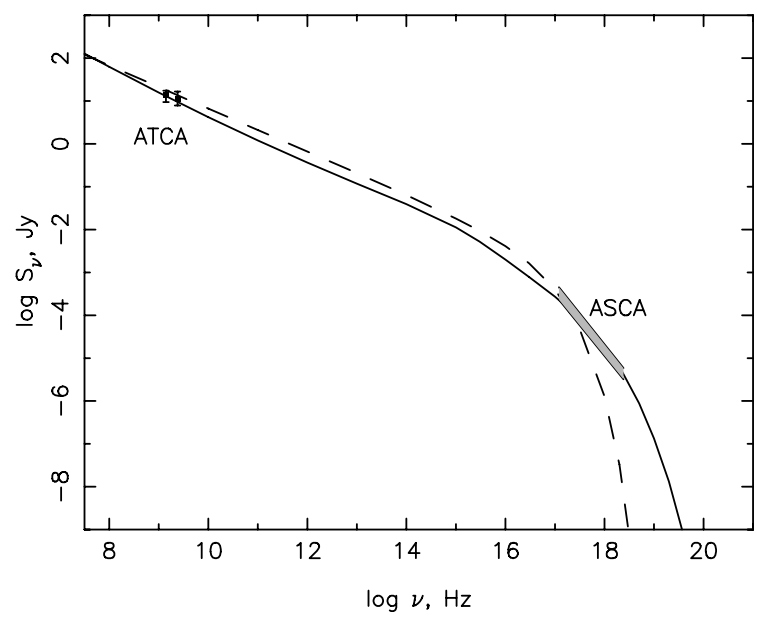

Fig. 4. Synchrotron photon flux density as a function of frequency. The solid line corresponds to the high-injection model, the dashed line corresponds to the test-particle approximation. The ATCA radio data and ASCA X-ray data (Aharonian et al. 2006) are shown.

accelerated nuclear CRs do not produce any significant shock modification and therefore also no magnetic field amplification. This corresponds to the test particle limit, and the low value $B_{0}=5 \mu \mathrm{G}$ was adopted for this case, for which synchrotron cooling is negligible. There are two meager differences in the synchrotron spectra, corresponding to these two scenarios. The high-injection scenario leads to a steep radio spectrum $S_{v} \propto v^{-\alpha}$ with power law index $\alpha=0.62$ whereas in the test particle case $\alpha=0.5$. The quality of the existing radio data does not allow to distinguish these two scenarios. On the other hand the two spectra behave essentially different at X-ray frequencies $v \gtrsim 10^{18}$. They demonstrate that only in the high-injection case case with its high, amplified magnetic field value $B_{\mathrm{d}} \approx 100 \mu \mathrm{G}$ the spectrum $S_{v}(v)$ has a smooth cutoff consistent with the experiment (see Fig. 4). In the test particle case the spectrum $S_{v}(v)$ has too sharp a cutoff to be consistent with the observations.

The properties of small scale structures of SNR RX J1713.73946 seen in X-rays by Uchiyama et al. (2003), and in particular by Hiraga et al. (2005), provide even stronger evidence that the magnetic field inside the SNR is indeed considerably amplified (see Sect. 2.1).

In order to find out if the filamentary structure found by (Hiraga et al. 2005) is indeed consistent with the high-injection case we show in Fig. 5 the projected radial brightness profile

$J(\epsilon, \rho) \propto \int \mathrm{d} x q\left(\epsilon, r=\sqrt{\rho^{2}+x^{2}}, x\right)$,

calculated for the X-ray energy $\epsilon=\epsilon_{v}=1 \mathrm{keV}$. Here $q(\epsilon, r)$ is the spectral luminosity of the nonthermal emission with photon energy $\epsilon$. The integration is performed along the line of sight $x$. For high injection the theory predicts the peak of the emission just behind the shock front with thickness $\Delta \rho / R_{\mathrm{S}} \approx 10^{-2}$ that corresponds to an angular width $\Delta \psi \approx 0.4^{\prime}$. This width is significantly thinner than the observed width of $\Delta \psi \approx 2.2^{\prime}$. However, one should take into account the smoothing procedure with which the data were obtained/presented. Instead of the actual profile $J_{\gamma}(\rho)$ it gives a broadened profile

$J^{\prime}(\rho)=\int_{-\infty}^{\infty} \mathrm{d} \rho^{\prime} G\left(\rho, \rho^{\prime}\right) J\left(\rho^{\prime}\right)$,

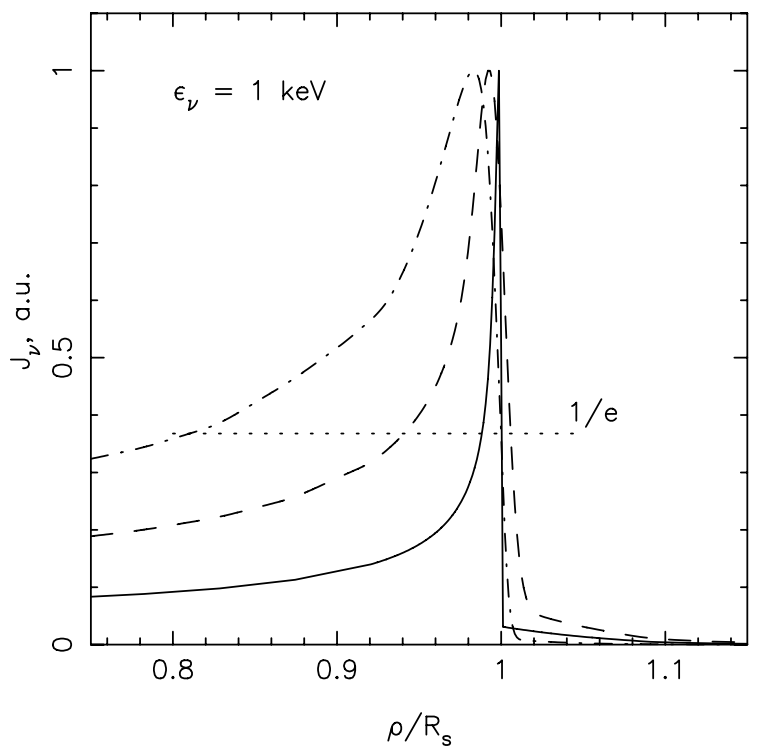

Fig. 5. Projected radial profile of the X-ray synchrotron emission for the energy $\epsilon_{v}=1 \mathrm{keV}$. The solid line corresponds to the high-injection model; the dashed line again represents the above profile, but smoothed to the resolution of the XMM-Newton data used in Hiraga et al. (2005); the dash-dotted line corresponds to the test-particle limit. For purposes of presentation all profiles are normalised to their peak values in this figure.

through the (Gaussian) point spread function

$G\left(\rho, \rho^{\prime}\right)=\left(\sigma_{\rho} \sqrt{2 / \pi}\right) \exp \left[-\left(\rho-\rho^{\prime}\right)^{2} /\left(2 \sigma_{\rho}^{2}\right)\right]$,

where $\sigma_{\rho}=\sigma_{\psi} d$, and $\sigma_{\psi}$ is the angular resolution of the instrument (in radians). If we apply such a point spread function with $\sigma_{\psi}=12.8^{\prime \prime}$ (corresponding to the smoothed XMM data) to the calculated profile, we obtain the broadened profile, which is shown in Fig. 5 by the dashed curve. It has a width $\Delta \rho / R_{\mathrm{S}} \approx 6.6 \times 10^{-2}$, or $\Delta \psi \approx 2^{\prime}$, and is consistent with the observational profile presented in Fig. 2 of Hiraga et al. (2005) which was discussed in Sect. 2.1.

In addition, we present in Fig. 5 the projected radial profile (dash-dotted line) which corresponds to the test particle limit. Since in this case the synchrotron losses are not relevant on account of the much lower magnetic field value $B_{\mathrm{d}}=20 \mu \mathrm{G}$, the peak of the emission is broader by a factor of five, inconsistent with the XMM observations.

Morphologically the remnant has a rather complex structure. This is particularly visible in the very bright western parts. Instead of a single, bright thin rim which should be identified with the actual position of the forward SNR shock, there are two nested, narrow arc-like rims (Cassam-Chenaï et al. 2004). The additional second inner bright rim cannot be associated within our model with a structure situated inside the remnant at the corresponding distance behind the forward shock. On the other hand Cassam-Chenaï et al. (2004) found a positive correlation between the X-ray brightness and the absorbing column density. It is possibly explained by assuming that all bright spots observed in X-rays lie just behind the forward shock, whose shape is significantly distorted due to distortions of the dense, sweptup shell. Such shell distortions can in turn can be expected because of inhomogeneities of the external ISM. In such a picture the outer rim, associated with the position of the forward SNR shock, is bright due to the projection effect, whereas the inner rim is bright because the corresponding part of the shock interacts with a denser medium. 


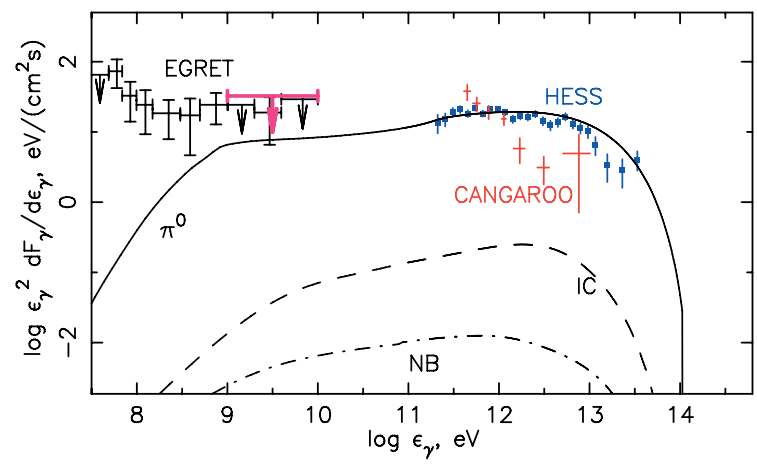

Fig. 6. Nonthermal bremsstrahlung (dash-dotted line) IC (dashed line) and $\pi^{0}$-decay (solid line) $\gamma$-ray spectral energy distribution as a function of $\gamma$-ray energy. The observed HESS (Aharonian et al. 2006) and CANGAROO (Enomoto et al. 2002) $\gamma$-ray fluxes and the EGRET spectrum of 3EG J1714-3857 (Reimer \& Pohl 2002) and the EGRET upper limit are shown as well.

Concentrating now on $\gamma$-ray energies the calculated nonthermal Bremsstrahlung, IC and $\pi^{0}$-decay $\gamma$-ray spectral energy distributions are shown in Fig. 6 together with the existing experimental data. (In this presentation the theoretical spectrum can be directly visually compared with the HESS results, cf. Fig. 20 of Aharonian et al. 2006.) According to the calculation, the hadronic $\gamma$-ray production exceeds the electron contribution by more than two orders of magnitude at all energies (Fig. 6).

In the context of the CANGAROO observations of RX J1713.7-3946, which covered only the northern part of the remnant (Enomoto et al. 2002), Butt et al. (2002) as well as Reimer \& Pohl (2002) had argued that the GeV emission from the nearby unidentified EGRET source 3EG J1714-3857 is either associated with the SNR or an upper limit to the $\gamma$-ray emission of RX J1713.7-3946. As one can see from Fig. 6 such an upper limit does not contradict our calculated spectrum of RX J1713.7-3946. This is even true for an extrapolation of the observed HESS spectrum in the test-particle approximation, with a spectral index of $\approx 2$ (Aharonian et al. 2006).

For energies $\epsilon_{\gamma}<3 \mathrm{TeV}$ the theoretical $\gamma$-ray spectrum is as hard as $\mathrm{d} F_{\gamma} / \mathrm{d} \epsilon_{\gamma} \propto \epsilon_{\gamma}^{-1.8}$, whereas for $\epsilon_{\gamma}>10 \mathrm{TeV}$ it has a smooth cutoff. We note that the $\gamma$-ray cutoff energy $\epsilon_{\gamma}^{\max } \approx 0.1 c p_{\max }$ is sensitive to the magnetic field strength $B_{\mathrm{d}}$, since the proton cutoff momentum has a dependence $p_{\max } \propto R_{\mathrm{s}} V_{\mathrm{s}} B_{\mathrm{d}}$ (Berezhko 1996). It is clearly seen from Fig. 6 that the calculated spectrum in a satisfactory way fits the HESS measurements.

Since the SN shock propagates through the shell with a rising gas density, the $\mathrm{TeV}$ emission is expected to increase with a rate of about $0.4 \% / \mathrm{yr}$, whereas the nonthermal X-ray emission goes down with a rate $0.1 \% / \mathrm{yr}$ due to the shock deceleration.

We note also that within our approach there is no need to suggest the interaction of the $\mathrm{SN}$ shock with the molecular cloud to be followed by a strong escape of high energy CRs, in order to explain the observed steepening of $\gamma$-ray spectrum at $\epsilon_{\gamma}>$ $1 \mathrm{TeV}$, as proposed by Malkov et al. (2005).

The projected radial $\gamma$-ray emission profile, calculated for the energy $\epsilon_{\gamma}=1 \mathrm{TeV}$, is presented in Fig. 7. As a result of the large radial gradient of the gas density and the CR distribution inside the SNR, the theoretically predicted radial profile of the TeV-emission is concentrated within a thin shell of width $\Delta \rho \approx 0.1 R_{\mathrm{s}}$. Due to the projection effect this width is seven times larger than the width of the 3-dimensional radial emissivity profile, which is as thin as $\Delta r \approx 0.01 R_{\mathrm{s}}$. Since the HESS instrument has a finite angular resolution we present in Fig. 7 also the

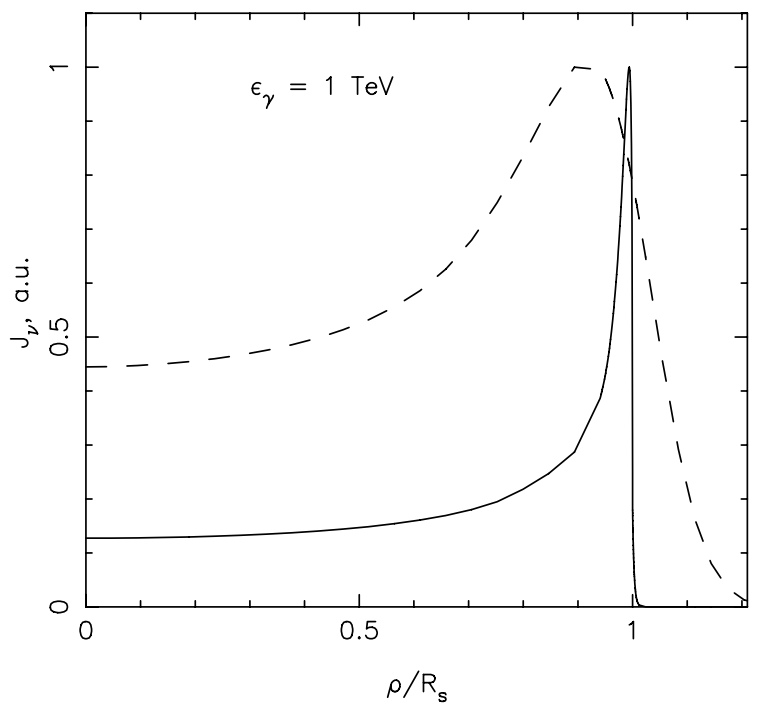

Fig. 7. The $\gamma$-ray emissivity for $\gamma$-ray energies $\epsilon_{\gamma}=1 \mathrm{TeV}$ as a function of angular radial distance. The calculated radial profile is given by the solid line; the dashed line represents the calculated profile smoothed with a Gaussian point spread function of the width $\sigma_{\psi}=0.05^{\circ}$. For purposes of presentation both profiles are normalised to their peak values in this figure.

modified radial profile smoothed with the Gaussian point spread function with $\sigma_{\rho}=\Delta \rho=0.08 R_{\mathrm{s}}$, that corresponds to the angular resolution $2 \sigma_{\psi}=0.1^{\circ}$. As shown by Fig. 7 the smoothed radial profile of the TeV-emission is much broader and - what is most interesting - it is characterised by a maximum to minimum intensity ratio $J_{\gamma}^{\max } / J_{\gamma}^{\min }=2.2$. Such a ratio is consistent with the HESS measurement (Aharonian et al. 2006) which obviously gives only a lower limit to the sharpness of the $\gamma$-ray profile. We conclude that the broad radial profile of the $\mathrm{TeV}$-emission with $J_{\gamma}^{\max } / J_{\gamma}^{\min } \approx 2$ measured by the HESS instrument is indirect evidence that the actual radial profile is significantly sharper, with a higher ratio $J_{\gamma}^{\max } / J_{\gamma}^{\min }>2.2$. Also, comparing with the radial X-ray profile in Fig. 5, the similarity of the radial profiles of the X-ray, as measured by ASCA and the radial $\mathrm{TeV} \gamma$-ray profiles, as obtained by HESS, must be seen as a consequence of the finite angular resolutions of these instruments. In reality, the $\mathrm{X}$-ray profiles should be narrower than the $\gamma$-ray profiles due to the synchrotron losses.

\section{Discussion and summary}

The theoretical results presented here are fully consistent with a dominantly hadronic origin of the observed $\mathrm{TeV} \gamma$-rays. Beyond that they of course imply that the overwhelming contribution to the nonthermal pressure comes from accelerated nuclear particles. Despite the observational fact that the broadband emission is not fully spherically symmetric, with the brightness increasing from southeast to northwest, the quasi-spherical character of the X-ray rims on the western half of the SNR suggests the approximate validity of a spherically symmetric model, with clear limitations due to the non-spherical nature of the magnetic field configuration which are to be corrected for.

The true difficulty for the theoretical description is therefore not the complex geometry of the distribution of molecular gas and X-ray absorbing column density, but rather the fact that several key parameters of this source are either not known or poorly constrained. This already concerns the distance and age of the object. We follow present consensus which puts the distance at 
$1 \mathrm{kpc}$ and the age to about 1600 years. We also concur with other authors and argue that the primary explosion must have been a type II/Ib SN event with a massive progenitor star whose mass loss in the main sequence phase created a hot wind bubble in a high-density environment. However, at present these remain assumptions, as well-founded as they are. The solution for the overall remnant dynamics then yields the value for the expansion velocity of the outer shock, given the total mechanical energy $E_{\mathrm{sn}}$ released in the explosion. To obtain a consistent solution for the broadband nonthermal emission we chose $E_{\mathrm{sn}}=1.8 \times 10^{51} \mathrm{erg}$ which is already on the high side, also for core collapse SNRs with initial main sequence progenitor masses between 15 and $20 M_{\odot}$ (Hamuy 2003). Therefore this particular parameter value is limited from above.

The most difficult aspect is the absence of a reliable radio synchrotron spectrum. In other SNRs, such as SN 1006, Cas A and Tycho's supernova, the deviation of the radio photon index $\alpha$ from its test particle value $\alpha=0.5$ allows the determination of the injection rate of the dominant nuclear particles into the acceleration mechanism at the forward SNR shock, and thereby the nonlinear modification of the shock and of the acceleration process. Fitting then the overall synchrotron spectrum, from radio to $\mathrm{X}$-ray frequencies, at the same time the effective magnetic field inside the SNR can be determined with reasonable accuracy.

In the absence of a radio spectral index we have estimated the strength of the effective field by analysing the observed width of an X-ray filament in a smoothed radial profile which clearly indicates the position of the outer shock. The result is a lower limit to the effective magnetic field strength of about $70 \mu \mathrm{G}$. Taking a value of about $130 \mu \mathrm{G}$ to be representative for the postshock value over $80 \%$ of the remnant's outer surface, we can calculate the IC $\gamma$-ray emission from the known X-ray synchrotron emission. Its negligible contribution - at the present epoch - to the observed TeV $\gamma$-ray flux, similar to that from nonthermal Bremsstrahlung, shows that SNR RX J1713.7-3946 must be a hadronic $\gamma$-ray source. This is our first major conclusion.

The above magnetic field strength is clearly amplified with respect to its expected value at the inner border of the hot wind bubble. Theoretically this amplification can only come from an efficiently accelerated nuclear particle component. And indeed, choosing as a consequence a proton injection rate similar to those determined for the other young SNRs mentioned above, it is possible to consistently describe the entire nonthermal broadband spectrum over 19 orders of magnitude in photon energy, including now the observed $\pi^{0}$-decay $\gamma$-ray spectrum. This is our second important conclusion, and it is far from trivial. On the other hand, one could argue that this remarkable result could be achieved because the observational constraints are not as numerous as they would be in the presence of a complete multi-wavelength knowledge. It therefore remains to justify the high nuclear injection rate to positively predict the amplitude of the $\gamma$-ray flux. A reasonably good radio spectrum could achieve this. The possibility of measuring a good thermal X-ray spectrum seems very limited from the known XMM-Newton results.

In the following we shall give a further going argument which is based on the physical idea that the magnetic field amplification is driven by the gradient of the CR pressure upstream of the outer shock. In fact, for all the thoroughly studied young SNRs mentioned above, the ratio of magnetic field energy density $B_{0}^{2} / 8 \pi$ in the upstream region of the shock precursor to the CR pressure $P_{\mathrm{c}}$ is about the same. Within an error of about 50 percent we have

$B_{0}^{2} /\left(8 \pi P_{\mathrm{c}}\right) \approx 5 \times 10^{-3}$.

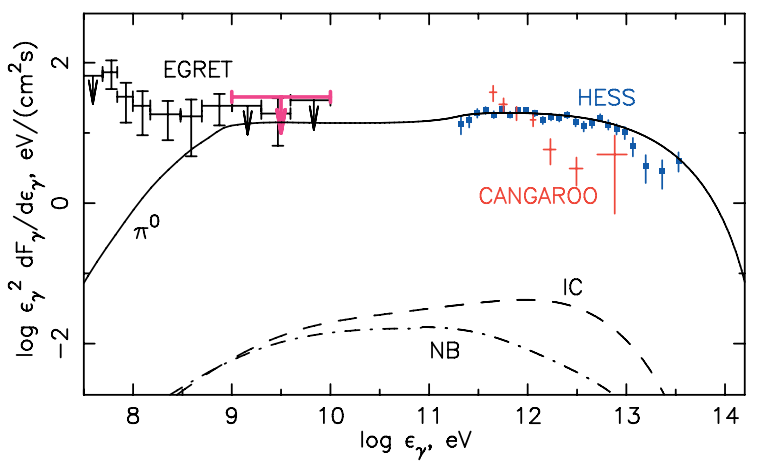

Fig. 8. Nonthermal Bremsstrahlung (dash-dotted line), IC (dashed line) and $\pi^{0}$-decay (solid line) $\gamma$-ray spectral energy distributions as a function of $\gamma$-ray energy for the case, where Eq. (15) is imposed as an additional constraint. The following parameters have been modified compared to those on which Fig. 6 is based: $E_{\mathrm{sn}}=2.5 \times 10^{51} \mathrm{erg}$, $M_{\mathrm{ej}}=5 M_{\odot}, B_{0}=50 \mu \mathrm{G}, \eta=10^{-4}$, which give rise, at the current epoch, to the values: $V_{\mathrm{s}}=1651 \mathrm{~km} \mathrm{~s}^{-1}, \sigma=4.93$, and $\sigma_{\mathrm{s}}=3.71 . N_{\mathrm{g}}$ was chosen as $N_{\mathrm{g}}=1.76 \mathrm{~cm}^{-3}$, in order to be able to visually compare the form of the resulting hadronic $\gamma$-ray energy spectrum with the observations. The data plotted are the same as in Fig. 6.

Requiring now this semi-empirical relation to hold also for SNR RX J1713.7-3946, we can ask ourselves, what magnetic field strength, larger than the lower limit which we could determine from the XMM observations, can reproduce the observations. For this consideration we assume in addition that the total mechanical explosion energy $E_{\mathrm{sn}}$ is not larger than $2.5 \times 10^{51} \mathrm{erg}$, i.e. that RX J1713.7-3946 is not an outlier in the distribution of core collapse explosion energies with main sequence progenitor masses below $20 M_{\odot}$ (compare also Hamuy 2003). Together with other parameters (see Fig. 8), roughly equal to those given in Sect. 2, even this large value of $E_{\mathrm{sn}}$ can reproduce the observations, not counting here the $\gamma$-ray observations. The associated proton injection rate is then also determined. Finally, the resulting theoretical hadronic $\gamma$-ray spectrum must be compared to the $\mathrm{TeV}$ observations. Obviously the above additional constraints make it more difficult to fulfil the sum total of the constraints. In addition, the relevant gas density at the bubble wall remains poorly constrained. However, the ratio $E_{\mathrm{c}} / E_{\mathrm{sn}}$ of nuclear CR energy to mechanical explosion energy is now limited from below.

In a limited coverage of the entire parameter space it turns out that (i) $B_{\mathrm{d}} \approx 250 \mu \mathrm{G}$ (ii) that the ion injection rate $\eta=10^{-4}$ is a factor of 3 smaller than assumed in the previous section, and (iii) that the total CR energy inside the remnant is $E_{\mathrm{c}}=$ $0.22 f_{\mathrm{re}} E_{\mathrm{sn}} \approx 10^{50} \mathrm{erg}$. It will grow in the spherically symmetric case to about $50 \%$ of $E_{\mathrm{sn}}$ in another few thousand yrs, in general agreement with earlier studies of wind-SNe (Berezhko \& Völk 2000a). Choosing a present-day pre-shock density which is about 50 percent higher than assumed in Sect. 2, one can even fine-tune the amplitude factor of the hadronic $\gamma$-ray flux to the observed amplitude, in order to compare with the observed spectral form. The result is shown in Fig. 8. It demonstrates that the new solution is less nonlinearly modified than before. However, the observed part of the spectrum is quite well reproduced. It is quite clear from Figs. 6 and 8 that measurements of the $\gamma$-ray spectrum at low energies $\epsilon_{\gamma} \lesssim 10 \mathrm{GeV}$, which can be done with the $\gamma$-ray space telescope GLAST in the future, could provide very useful information about the degree of SNR shock modification.

We conclude that the present observational knowledge of SNR RX J1713.7-3946 can be interpreted by a source which 
ultimately converts about $10 \%$ of the mechanical explosion energy into nuclear CRs and that the observed high energy $\gamma$-ray emission of SNR RX J1713.7-3946 is of hadronic origin.

Acknowledgements. This work has been supported in part by the Russian Foundation For Basic Research (grant 03-02-16524). E.G.B. acknowledges the hospitality of the Max-Planck-Institut für Kernphysik, where part of this work was carried out. H.J.V. is grateful to the other members of the HESS collaboration for numerous discussions about RX J1713.7-3946. The authors thank L. Ksenofontov for his assistance in the preparation of this paper.

\section{References}

Abbott, D. C. 1982, ApJ, 263, 723

Aharonian, F. A., Akhperjanian, A. G., Aye, K.-M., et al. (HESS Collaboration) 2004, Nature, 432,75

Aharonian, F. A., Akhperjanian, A. G., Aye, K. M., et al. (HESS Collaboration) 2006, A\&A 449,223

Bell, A. R. 1978, MNRAS, 182, 147

Bell, A. R., \& Lucek, S. G. 2001, MNRAS, 321, 433

Bell, A. R. 2004, MNRAS, 327, 433

Berezhko, E. G., Elshin, V. K., \& Ksenofontov, L. T. 1996, JETPh, 82,

Berezhko, E. G. 1996, Astropart. Phys., 5, 367

Berezhko, E. G., \& Völk, H. J. 1997, Astropart. Phys., 7, 183

Berezhko, E. G., \& Ksenofontov, L. T. 1999, JETPh, 89, 391

Berezhko, E. G., \& Völk, H. J. 2000a, A\&A, 357, 183

Berezhko, E. G., \& Völk, H. J. 2000b, Astropart. Phys., 14, 201

Berezhko, E. G., Ksenofontov, L. T., \& Völk, H. J. 2002, A\&A, 395, 943

Berezhko, E. G., Ksenofontov, L. T., \& Völk, H. J. 2003a, A\&A, 412, L11

Berezhko, E. G., Pühlhofer, G., \& Völk, H. J. 2003b, A\&A, 400, 971

Berezhko, E. G., \& Völk, H. J. 2004a, A\&A, 419, L27

Berezhko, E. G., \& Völk, H. J. 2004b, A\&A, 427, 525

Berezhko, E. G. 2005, Adv. Space Res., 35, 1031
Blandford, R. D., \& Ostriker, J. P. 1978, ApJ, 221, L29

Butt, Y. M., Torres, D. F., Combi, J. A., et al. 2002, Nature, 418, 499 Cassam-Chenaï, G., Decourshelle, A., Ballet, J., et al. 2004, A\&A, 427, 199 Chevalier, R. A. 1977, Ann. Rev. Astrophys., 15, 175

Chevalier, R. A. 1982, ApJ, 258, 790

Chevalier, R., \& Liang, P. 1989, ApJ, 344, 332

Ellison, D. C., Slane, P., \& Gaensler, B. M. 2001, ApJ, 563, 191 Enomoto, R., Tanimori, T., Naito, T., et al. 2002, Nature, 416, 823 Fukui, Y., Moriguchi, Y. Tamura, K., et al. 2003, PASJ, 55, L61 Hamuy, M. 2003, ApJ, 582, 905

Hiraga, J. S., Uchiyama, Y., Takahshi, T., \& Aharonian, F. A. 2005, A\&A, 431, 953

Jones, E. M., Smith, B. W., \& Straka, W. C. 1981, ApJ, 249, 185 Koyama, K., Kinagasa, K., Matsuzaki, K., et al. 1997, PASJ, 49, L7

Ksenofontov, L. T., Berezhko, E. G., \& Völk, H. J. 2005, A\&A, 443, 973

Lazendic, J. S., Slane, P. O., Gaensler, B. M., et al. 2004, ApJ, 602, 271

Lucek, S. G., \& Bell, A. R. 2000, MNRAS, 314, 65

Malkov, M. A., Diamond, P. H., \& Sagdeev, R. Z. 2005, ApJ, 624, L37

McKenzie, J. F., \& Völk, H. J. 1982, A\&A, 116, 191

Moriguchi, Y., Tamura, K., Tawara, Y., et al. 2005, ApJ, 631, 947

Muraishi, H., Tanimori, T., Yanagita, S., et al. 2000, A\&A, 374, 895

Pfeffermann, E., \& Aschenbach, B. 1996, in Roentgenstrahlung from the Universe, ed. H. H. Zimmermann, J. Trümper, \& H. Yorke (MPE Rep. 263; Garching: MPE), 267

Reimer, O., \& Pohl, M. 2002, A\&A, 390, L43

Slane, P., Gaensler, B. M., Dame, T. M., et al. 1999, ApJ, 525, 357

Uchiyama, Y., Aharonian, F. A., \& Takahshi, T. 2003, A\&A, 400, 567

Völk, H. J. 2003, in Frontiers of Cosmic Ray Science, Proc. 28th ICRC, Tsukuba, ed. T. Kajita, Y. Asaoka, K. Kawachi, Y. Matsubara, \& M. Sasaki (Tokyo, Japan: Universal Press), Inc., Invited papers, 8, 29 [arXiv: astro-ph/0312585]

Völk, H. J., \& McKenzie, J. F. 1981, in Proc. 17th ICRC, Paris, 9, 246

Völk, H. J., Berezhko, E. G., Ksenofontov, L. T., \& Rowell, G. P. 2002, A\&A, 396, 971

Völk, H. J., Berezhko, E. G., \& Ksenofontov, L. T. 2003, A\&A, 409, 563

Völk, H. J., Berezhko, E. B., \& Ksenofontov, L. T. 2005, A\&A, 433, 229

Wang, Z. R., Qu, Q.-Y., \& Chen, Y. 1997, A\&A, 318, L59

Weaver, R., McCray, R., Castor, J., et al. 1977, ApJ, 218, 377 